\title{
LA PINTURA COMO INHIBIDOR DE CORROSIÓN EN LAS ESTRUCTURAS DE ACERO ESPECIALMENTE EXPUESTAS Y SU RENDIMIENTO
}

\author{
(THE PAINTING AS CORROSION INHIBITOR IN STEEL STRUCTURES SPECIALLY \\ EXPOSED, AND ITS OUTPUT)
}

Jesús González Martín, Prof. de Construcciones Arquitectónicas de la EUAT de Madrid-U. P. M.

ESPAÑA

Fecha de recepción: 27-IV-99

\section{RESUMEN}

Se plantea un sistema de protección contra la corrosión en las estructuras de acero, a través de la prevención, mediante un sistema a base de pintura y la forma de aplicarla.

\section{SUMMARY}

A protection system is here exposed, against the corrosion in steel structures, preserving them through a painting process and the right application of this painting.
Muchos de los materiales de construcción que utilizamos nos llegan después de haber sufrido una transformación por parte del hombre, que, desde su estado natural y mediante un aporte energético, los convierten en otro material. Sin embargo, esta energía, artificialmente comunicada, tiende a perderse para volver a su estado natural, completándose así un ciclo, en el cual su energía libre es menor. Es el envejecimiento natural, relacionado con lo que, recientemente, se tiende a definir como una duración de vida en servicio limitada. Este tema suscita cada vez mayor interés, tanto por razones económicas, para obtener el máximo rendimiento de un material, aplicándole el mínimo de energía, como por razones técnicas de diseño, utilizando el máximo de las características del material con el grado de seguridad suficiente.

En las estructuras de acero el envejecimiento natural es lo que desemboca en su corrosión. Al estudiar sus principios, hay que manejar continuamente los fundamentos básicos de la electroquímica, ya que la corrosión es, en esencia, una combinación de procesos eléctricos y químicos.

Se puede estar familiarizado con las manifestaciones de la electricidad y de la química, aunque no se conozca bien que tales fenómenos son producidos por un continuo flujo de electrones. Para obtener un amperio de corriente a partir de una pieza metálica de cualquier tipo, deben pasar un cierto número de electrones cada segundo. Este número puede escribirse como $6 \times 10^{18}$, o sea, 6 trillones de electrones. La electricidad no es otra cosa que una forma de energía, que puede manifestarse como trabajo, calor o luz.

\section{Corrosión atmosférica}

El acero siempre ha de protegerse, para evitar la oxidación que se produce por el contacto del material con la atmósfera, por acción conjunta del agua y el oxígeno del aire. La corrosión se presenta en razón directa a la cantidad de humedad que aparece en la atmósfera. Al agua y al oxígeno hay que añadir otros elementos contaminantes que favorecen la corrosión, tales como humos, gases, salinidad, etc. Para proteger al acero contra la corrosión basta con aislarlo del contacto con la atmósfera y el agua, bien sea mediante una capa fina de otro metal o biē mediante pinturas. Este último es el medio más fácil y v̄ersátil, pues puede aplicarse en cualquier momento de la vida de la pieza metálica y se adapta a cualquier forma del metal. 


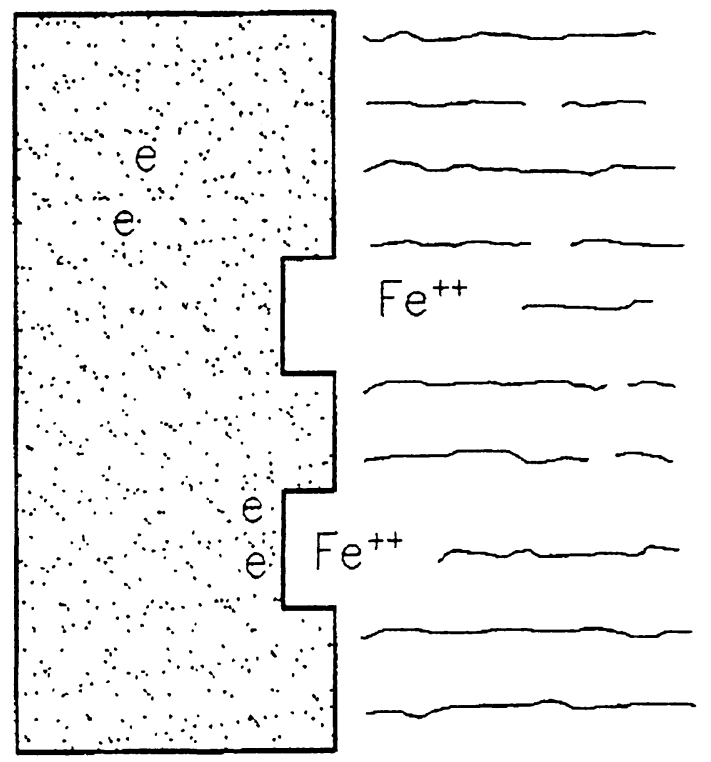

El acero pasa a la solución $\mathrm{Fe}^{++}$, liberando 2 electrones.

\section{Procedimientos para frenar o retrasar el envejeci- miento natural del acero. La protección catódica}

La protección catódica se basa en la transformación en cátodos de las estructuras a proteger mediante un circuito eléctrico controlado. Fue Sir Humphrey Davy el primero que, en 1824, sugirió que la corrosión de un metal podría evitarse uniéndolo a otro menos noble, proponiendo, así, proteger los forros de cobre.

La característica esencial de la protección catódica es la creación de una corriente eléctrica de sentido opuesto a la corriente de corrosión, conteniendo entonces las reacciones anódicas, que no pueden tener lugar en el metal a proteger al no poder llevarse a cabo la liberación de electrones. Toda la corriente se invierte en producir reacciones catódicas que tendrán lugar a lo largo de toda la superficie protegida, con la consiguiente reducción de iones hidrógeno o del oxígeno disuelto. Como consecuencia, el electrólito se vuelve alcalino en la proximidad de la estructura protegida, lo que puede resultar perjudicial para las películas de pintura, por cuanto el álcali puede saponificar todo tipo de pinturas que contengan aceite. Por otra parte, el desprendimiento de burbujas de hidrógeno llega a causar ampollas, todo lo cual desembocará en el desprendimiento de la película de pintura. El flujo de electrones se consigue también «imprimiendo» una corriente procedente de una fuente adecuada: una pila, una dinamo o un rectificador.

\section{El adecuado diseño de las estructuras garantiza un buen trabajo de protección con pintado y un correcto mantenimiento}

Como se ha mencionado anteriormente, las estructuras de acero, normalmente, se tienen que proteger contra la corrosión, lo cual puede realizarse de muchas maneras. Comencemos por considerar los problemas antes de que ocurran, es decir, preveamos un sistema de pintura.

La clave para la durabilidad y la eficacia de un sistema de pintado reside en la preparación de la superficie y la aplicación de la pintura, además de añadir el diseño y la construcción de la estructura a proteger.

Vamos a enumerar algunos problemas típicos previos relativos a la geometria y aspecto de la pieza. Deberá tener, dentro de lo posible, un número muy reducido de cantos vivos, cavidades, cordones de soldadura rugosos, etc., en donde la corrosión sea más susceptible de tener lugar o que sean difíciles, por no decir imposibles, de poder limpiar y pintar adecuadamente. Este estudio llevará a llamar la atención sobre un cierto número de aspectos, que, en caso de no respetarse, se deberán, al menos, manifestar, ante el constructor y la empresa aplicadora, antes de que la preparación de superficie comience. De esta forma se logrará minimizar la corrosión y las necesidades de mantenimiento $\mathrm{y}$, consecuentemente, se optimizará la durabilidad de los sistemas de pintado anticorrosivos.

Los aspectos más relevantes son:

1.- Planeidad de las superficies. Son preferibles las superficies planas que las irregulares.

2.- Son preferibles las superficies romas o curvas. En lugar de los cantos vivos, cualquier forma de aristas vivas tiende a reducir el espesor de la película de pintura. Ésta tiende a fluir, alejándose de la arista y, por consiguiente, serán preferibles los cantos redondeados, por su mayor susceptibilidad de ser pintados de forma uniforme.

Este defecto puede remediarse aplicando la pintura adicional directamente sobre el canto, hasta obtener una cobertura completa y conseguir, de esta forma, una capa extra de pintura en el canto, cuando ello sea posible.

Cuando exista condensación, hay una tendencia a que la humedad se acumule y fluya a lo largo del canto, como sucede, por ejemplo, en el caso de vigas en doble $\mathrm{T}$.

Se evitararán las supeficies rugosas e irregulares y que produzcan abrasión entre sí.

Asimismo, se evitarán las superficies elásticas, o bien, en su caso, habrá que protegerlas con pinturas especiales, que no se cuarteen y pierdan adherencia.

3.- Deberán evitarse las cavidades y grietas, por la retención de humedad. Con el aporte y el efecto calefactor del sol, la humedad se evaporará en los casos normales. Sin embargo, algunos ensamblajes y juntas impiden o retardan 

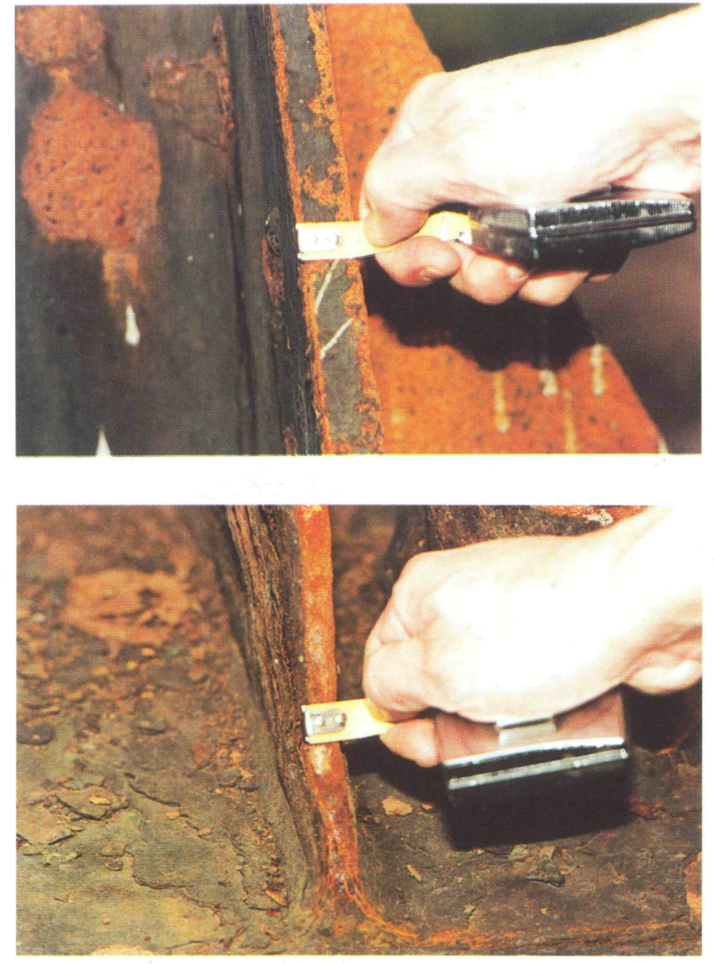

Pérdida de espesor por la corrosión. En la fotografia superior, $2 \mathrm{~cm}$; en la inferior, sólo 1 .

esta evaporación. Naturalmente, estas situaciones deberán eliminarse donde sea posible la aparición de una grieta.

4.- Soldaduras. Los problemas principales, en relación con la soldadura, son:

. Los cordones rugosos e irregulares, deberán alisarse y recibir doble capa de pintura para obtener una protección efectiva.

. Las escorias de soldadura pueden ser fuertemente alcalinas e higroscópicas. Si permanecen sobre el cordón absorberán humedad y crearán puntos en los que deben esperarse fallos de pintado. Por consiguiente, deberán eliminarse cuidadosamente.

. Las uniones de aristas deberán soldarse. La soldadura continua será preferible a las soldaduras por puntos. Las soldaduras por solape deben evitarse.

. La soldadura por puntos se usa ampliamente en la construcción, principalmente en los casos en los que no se considere necesario un cordón continuo. Estas soldaduras son vulnerables desde el punto de vista de la corrosión y no pueden limpiarse y pintarse adecuadamente. Por lo tanto, los equipos y estructuras que hayan de usarse en ambientes húmedos y corrosivos deberán tener todas sus uniones soldadas mediante cordones continuos.
. Soldadura por solape. En este caso el cordón es continuo en la cara exterior solamente, dejando las planchas simplemente solapadas en el interior. Fácilmente se ve que este hecho crea grietas que son imposibles de proteger adecuadamente.

5.- Se deberá permitir el escape de agua de todas las superficies. Los perfiles estructurales pueden constituir, por ellos mismos, zonas o puntos de retención de humedad.

En este caso, el problema se resolverá practicando agujeros de drenaje, allá donde sea posible, siempre que este agua no se vierta, a su vez, en elementos estructurales en posición inferior, ya que ello resultaría, igualmente, peligroso.

El goteo desde cubiertas de hormigón y puentes sobre elementos de acero inferiores, debe evitarse adecuadamente.

Cuando se empleen perfiles en U, se acumulará una cantidad considerable de agua de lluvia sobre la superficie expuesta y deberá controlarse su drenaje mediante un sistema adecuado.

6.- Las cartelas para andamiaje y otros montajes temporales para la fase de trabajos, deberán retirarse y los lugares donde se hallaban unidos, aislarse.

7.- Facilidad de mantenimiento. La mayoría de los recubrimientos tienen una vida corta, si se la compara a la propia de la estructura. De esta forma, es muy difícil evitar que tengan que hacerse repintados de mantenimiento a intervalos determinados.

- Si el mantenimiento no puede llevarse a cabo por imposibilidad física de alcanzar la superficie del acero, nos hallamos en una situación difícil. De esta forma, las juntas y fijaciones deberán disponerse de forma que nos proporcionen un trabajo lo más fácil posible.

. Este hecho evitará la acumulación de suciedad y humedad, al tiempo que facilita el acceso para mantenimiento.

....Y, como conclusión: un diseño adecuado, tomando en cuenta la corrosión, eliminará la mayoría de los problemas de mantenimiento posterior

\section{Proceso de pintado}

\section{a.- Limpieza de sales y suciedad}

Todo tipo de sales solubles, incrementará layelocidad de corrosión, porque proporcionan más iones que transportan la corriente eléctrica a través del electrólito. 
Si estos iones se encuentran debajo de la película de pintura, contribuyen a la formación de ampollas, debido al principio de «ósmosis», que es uno de los métodos naturales de restablecer el equilibrio. Cuando la concentración de sal es más elevada en uno de los lados de una membrana permeable, como lo es cualquier película de pintura, se produce un flujo de agua pura desde el otro lado de la membrana hasta que la concentración de sal en ambos lados se encuentra en el mismo nivel. Bajo circunstancias críticas puede ser tan necesario comprobar la ausencia de sales solubles y partículas de polvo, como lo es asegurarse de que el óxido y la calamina han sido eliminados hasta el grado de limpieza especificado para un trabajo particular.

Debe tenerse siempre presente que la película seca de una pintura es, más o menos, microporosa o permeable, por lo que dejará pasar algo de aire y humedad, que llegará a ponerse en contacto con el acero. Para disminuir al máximo esta permeabilidad basta con aumentar el grosor de película total, aplicando mayor número de capas, utilizando pinturas especiales denominadas "de capa gruesa" y eligiendo las resinas más impermeables.

Además de esta permeabilidad, asociada a la propia composición de la pintura (depende del aglutinante, de la proporción de pigmentos, etc.) hay que contar con las variaciones de grosor de película que se producen como consecuencia del sistema de aplicación, del tipo de pintura y de la forma del objeto que se quiere pintar. Por ejemplo, en los fondos de las huellas o marcas de la brocha hay menos grosor $\mathrm{y}$, por tanto, más permeabilidad que en las crestas, por eso se recomienda dar varias capas y cruzarlas, para que los valles y las crestas de unas se compensen con las de otras.

Teniendo en cuenta, pues, que es relativamente probable que, por una u otra causa, llegue la humedad (acompañada de sus contaminantes) a entrar en contacto con la superficie del acero y corroerlo por debajo de la capa de pintura, se utiliza el recurso de que la primera capa o imprimación contenga pigmentos anticorrosivos, el más conocido de los cuales es el minio de plomo, aunque también existen otros, como el silicato o cromato de zinc, el polvo de zinc, etc. Estos pigmentos tienen la propiedad de que al estar presentes en la zona de contacto de la humedad con el acero, inhiben o impiden la oxidación por un mecanismo de acción electroquímica.

\section{b.-Eliminación de calamina}

$\mathrm{Al}$ acero, después de su fabricación inicial, se le dan distintas formas (perfiles, planchas, láminas, redondos), mediante dos procedimientos conocidos: por laminación en caliente o laminación en frío. El primero, da origen a la mayoría de las formas que nos encontramos en construcción y se caracteriza porque produce siempre una costra de acero"quemado", oxidado por el contacto con oxígeno a alta temperatura, que se denomina "cascarilla

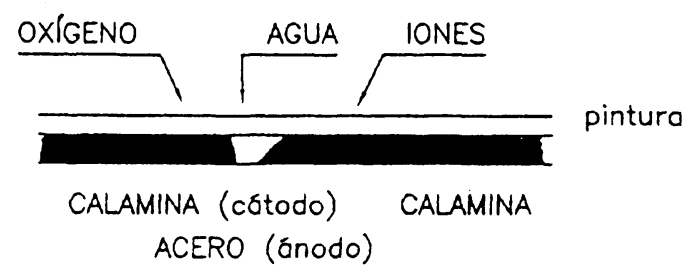

AMPOLLAMIENTO

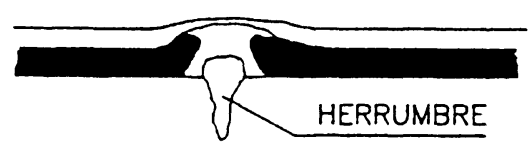

CUARTEAMIENTO

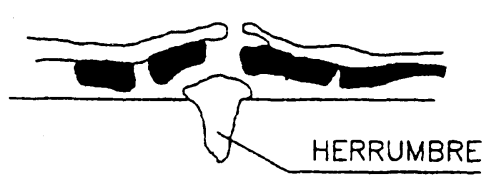

Eliminación de calamina.

de laminación" o "calamina". Esta costra dura está compuesta por óxidos de hierro y tiene una gran facilidad para agrietarse, dejando pasar humedad; por ello, no sirve como protección del acero, actuando, por el contrario, como un impedimento para que la imprimación llegue hasta él. Debe eliminarse totalmente -mediante chorro de arena -o parcialmente, dejándola desmoronarse por efecto de la intemperie, cepillando luego a fondo para eliminar sus residuos. Aunque hay otros métodos para realizar esta eliminación de la cascarilla o del óxido residual, no entraremos en detalle por no ser éste el objetivo. A este respecto, recomendamos la lectura de la publicación del Instituto Nacional de Racionalización del Trabajo "Instrucciones para la preparación de las superficies que se han de pintar", en la que se trata este tema con mucha amplitud.

No debe olvidarse que es fundamental, para lograr una buena adherencia de la pintura, la eliminación de las manchas de grasas y aceites, que acostumbran a estar presentes en las superficies metálicas, debido a su mecanización y manipulación, lo cual se hace, por lo general, mediante trapos empapados en disolventes.

Este desengrase es especialmente importante en las superficies de acero laminado en frío, en las que constituye la impureza principal, puesto que en ellas no existe la costra de laminación.

Como hemos mencionado antes, la calamina agrava los problemas de corrosión y se recomienda su completa eliminación, por chorreado con arena, para obtener el máximo rendimiento de una pintura. 
Generalmente es una mala práctica pintar sobre calamina. No solamente se produce una adherencia deficiente, sino que se favorece el desarrollo de corrosiones por picadura. El rendimiento de las pinturas sobre superficies mal preparadas varía considerablemente y hay situaciones en las que el recubrimiento se descascarilla al cabo de los pocos meses, o incluso al cabo de unas semanas. La calamina es una capa constituida por óxidos de hierro que se forman durante el proceso de laminación del acero, formada por 3 capas de óxido de hierro de composición diferente; la cantidad y forma de cada una varía según las condiciones de laminación. La capa que se forma junto al acero acostumbra a ser porosa y la descomposición de la misma durante la exposición a la intemperie, tiene tendencia a socavar el resto de la película, al permitir la oxidación por debajo de la misma. Dado que la calamina no recubre uniformemente la superficie del acero, si se aplica una pintura por encima de ella, quedarán pequeñas zonas de acero desnudo entre la película de pintura y la calamina.

Una pintura no podrá evitar el cuarteamiento y el desprendimiento de la calamina, por lo que establecerán algunas pilas de corrosión. Si se produce alguna rotura local, pequeñas zonas de acero desnudo quedarán sujetas al ataque de grandes superficies catódicas. Dado que la picadura y oxidación prosigue, el desarrollo de herrumbre por debajo de los bordes de la calamina la romperá o incluso resquebrajará, produciéndose el desprendimiento de pequeños trozos de la misma. Por tanto, si la pintura ha sido aplicada directamente sobre la calamina y no ha habido adherencia con el acero, podemos esperar que se produzcan fallos en el recubrimiento cuando la calamina se rompa. Al proseguir el desarrollo de los depósitos de herrumbre de forma continua, la pintura se soltará, lo cual se evidenciará por la aparición de desprendimientos, ampollas y cuarteamientos y terminando con una eventual pérdida general de adherencia.

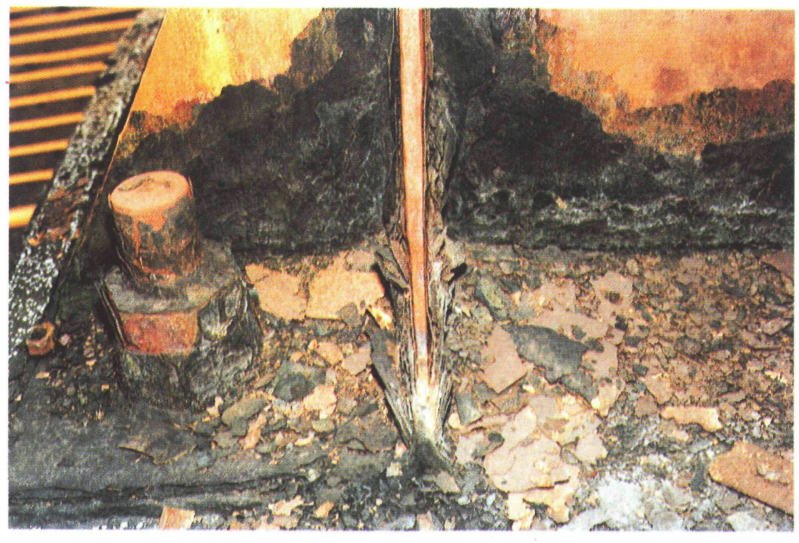

Se aprecia una pérdida de material por exfoliación.

Por tanto, es fácil de comprender que el único camino eficaz para preparar el acero, de cara al pintado, es la eliminación completa del óxido y la calamina.

\section{c.- Preparación de la superficie}

Otro factor importante, en relación con el nivel de adherencia del recubrimiento con el soporte, si se pretende obtener una pintura duradera, es extremar la preparación superficial.

Las superficies que estén contaminadas por aceites, grasas, etc., serán desengrasadas, utilizando detergentes o disolventes halogenados.

Las superficies con óxido, se tratarán conforme al cuadro que aparece a continuación, dependiendo del tipo de limpieza del soporte que se pretende cubrir y el tiempo de mantenimiento de repintado mínimo deseado, en función de las condiciones atmosféricas dominantes.

\begin{tabular}{|c|c|c|c|}
\hline & \multicolumn{3}{|c|}{ NORMAS } \\
\hline DESCRIPCIÓN & $\begin{array}{c}\text { BS-4232 } \\
\text { inglesa }\end{array}$ & $\begin{array}{c}\text { SSPC } \\
\text { EE UU }\end{array}$ & $\begin{array}{c}\text { SIS } \\
\text { sueca }\end{array}$ \\
\hline Chorreado a Metal blanco & $1^{\text {a } \text { Calidad }}$ & SSPC - SP 5 & Sa 3 \\
\hline Chorreado a Metal casi blanco & $2^{\text {a Calidad }}$ & SSPC - SP 10 & Sa 2 1/2 \\
\hline Chorreado comercial & $3^{\text {a Calidad }}$ & SSPC - SP 6 & Sa 2 \\
\hline Chorreado ligero & & SSPC - SP 7 & Sa 1 \\
\hline Limpieza mecánica & & SSPC - SP 3 & St 2 - 3 \\
\hline Limpieza manual & & SSPC - SP 2 & \\
\hline Limpieza con disolventes & & SSPC - SP 1 & \\
\hline Decapado & & SSPC - SP 8 & \\
\hline
\end{tabular}




\section{Descripción de los diferentes procedimientos de preparación de las superficies de acero}

- Chorreado abrasivo a metal blanco: el chorro se pasa sobre la superficie, a fin de eliminar la totalidad de la calamina, herrumbre y materias extrañas. Finalmente, la superficie se limpia, bien con una aspiradora o con aire comprimido, seco y limpio. Debe adoptar un color metálico uniforme.

La superficie metálica así chorreada, debe recibir la primera capa de imprimación tan pronto como sea posible, preferiblemente antes de transcurrida 1 hora y siempre antes de que la temperatura del metal descienda a menos de $3{ }^{\circ} \mathrm{C}$ por encima del punto de rocío y que se produzca reoxidación.

- Chorreado abrasivo a metal casi blanco: chorreado muy cuidadoso. La calamina, la herrumbre y las materias extrañas deben eliminarse de forma que sólo queden algunas trazas distribuidas uniformemente, tomando el aspecto de sombras en forma de manchas o franjas. Al final, la superficie debe limpiarse con una aspiradora, o bien con aire comprimido, seco y limpio.

. Chorreado comercial: chorreado cuidadoso. Casi toda la calamina, herrumbre y materias extrañas deben eliminarse. Finalmente, la superficie debe limpiarse con una aspiradora, aire comprimido seco y limpio o con un cepillo, limpio y seco.

- Chorreado ligero: consiste en el chorreado rápido de una superficie, usando un abrasivo fino. El objeto del chorreado ligero es comunicar rugosidad a una superficie, eliminar la contaminación de sales y otras impurezas de una superficie tratada con "shop primer" y expuesta largo tiempo a la intemperie, o eliminación de la herrumbre formada sobre superficies de acero previamente chorreadas.

. Rascado y cepillado mecánicos. rascado extremadamente cuidadoso (con una rasqueta metálica dura) y cepillado (con cepillo de alambre de acero). El rascado debe realizarse, primero, en un sentido y, posteriormente, en sentido perpendicular. La superficie se cepilla después vigorosamente, con un cepillo de alambre de acero.

Los productos mal adheridos se eliminan durante la operación, lo que permitirá comprobar el resultado. Finalmente, la superficie se limpia con una aspiradora, aire comprimido limpio y seco, o un cepillo, limpio y seco. Debe adquirir, entonces, un pronunciado brillo metálico. Una superficie tratada de esta forma, debe recibir la primera capa de imprimación tan pronto como sea posible, preferiblemente dentro de las 3 ó 4 horas siguientes y siempre antes de que la temperatura del metal descienda a menos de $3{ }^{\circ} \mathrm{C}$ por encima del punto de rocío y que se produzca reoxidación.

- Chorreado con agua: en los lugares donde está previsto realizar un chorreado abrasivo, las superficies pintadas pueden limpiarse, de cara a su repintado, mediante un chorro de agua a alta presión, con el resultado de que la pintura mal adherida, la contaminación de tipo químico, las acumulaciones de aceite y grasa, quedan eliminadas.

- Decapado: los decapantes son productos líquidos o semipastosos, cuyo componente principal es el cloruro de metileno (disolvente eficaz, de rápida evaporación y nula inflamabilidad). Lleva además otros disolventes: parafina, para frenar la evaporación; espesante, para que no escurra y se mantenga en las superficies verticales y otros aditivos que ayudan en la penetración.

El decapante ablanda, ahueca o desprende las capas de pintura en pocos minutos. Su acción se produce por una penetración a través de los puntos más débiles de la película de pintura, llegando hasta el soporte y, a partir de ahí, se difunde por la superficie, por debajo de la capa de pintura, despegándola total o parcialmente, según los casos.

Las más rápidas de despegar son las pinturas de gran tensión, como las sintéticas de secaje al horno y al aire, siendo su acción más lenta sobre viejas pinturas de aceite que, por su gomosidad, sobre todo cuando hay muchas capas, se ablandan y disuelven poco a poco, pero no se despegan en láminas enteras. En estos casos hay que insistir haciendo varias aplicaciones del producto decapante, ayudándole con la acción de la espátula.

\section{Rendimiento de las pinturas}

Se refiere al caso hipotético de superficies perfectamente lisas, sin pérdidas en la aplicación y admitiendo que el espesor de la película seca sea uniforme

\section{Rendimiento teórico}

Puede calcularse el rendimiento teórico de una pintura, a partir del tanto por ciento de sólidos en el volumen que tenga y del espesor especificado de la película seca, aplicando la siguiente fórmula:

\section{Rt: Rendimiento teórico}

Rt en $\mathrm{m}^{2} /$ litro $=\frac{10 \times \% \mathrm{~Sv}}{\mathrm{E}} ; \begin{aligned} & \mathrm{Sv}: \quad \text { Sólidos en volumen } \\ & \mathrm{E}: \text { Espesor de la película }\end{aligned}$ seca en micrómetros

$=$

Si se quiere el resultado en $\mathrm{m}^{2} / \overrightarrow{\mathrm{kg}}$, bastará dividirlo por el peso específico de la pintura. 


\section{Rendimiento práctico}

En la práctica, los consumos de pintura son siempre iuperiores, debido a los siguientes factores:

Juperficies que no están perfectamente lisas, a causa de rregularidades superficiales, restos de óxido y, sobretodo, 1 la rugosidad, obtenida debido al chorro de arena.

\i para simplificar suponemos que el arenado ha dejado jicos en forma de pirámides regulares de base cuadrada, zon lado igual a la altura (perfil de rugosidad), podría salcularse el volumen muerto de pintura extra necesaria Jara llenar los valles del perfil de rugosidad hasta el nivel zero, a partir de donde conviene contar el espesor eficaz de pintura.

Este volumen muerto aumenta considerablemente el zonsumo de pintura sobre una superficie rugosa con relación a una superficie lisa.

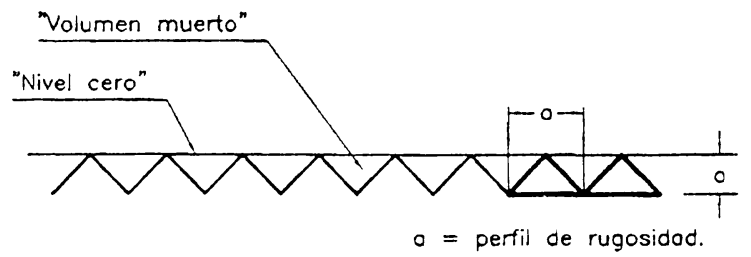

Relación entre perfil de rugosidad y consumo extra de pintura.

\section{Pérdidas de pintura en general}

Derrames y goteos durante la aplicación, los restos de pintura que quedan en los envases, mangueras, etc., también contribuyen a bajar el rendimiento.

De acuerdo con las consideraciones descritas anteriormente, pasamos a detallar, a continuación, unas recomendaciones de tipo general para deducir rendimientos prácticos de aplicación a partir del teórico.
El rendimiento práctico de aplicación puede estimarse multiplicando el rendimiento teórico por dos factores de corrección: factor debido a rugosidad y factor debido a la aplicación. Sus valores dependerán, en cada caso, de las condiciones que se dan en la práctica.

$$
\begin{aligned}
R p=R t \times f_{r} \times f_{a} ; \text { donde: } f_{r} & =\text { factor rugosidad } \\
f_{a} & =\text { factor de aplicación }
\end{aligned}
$$

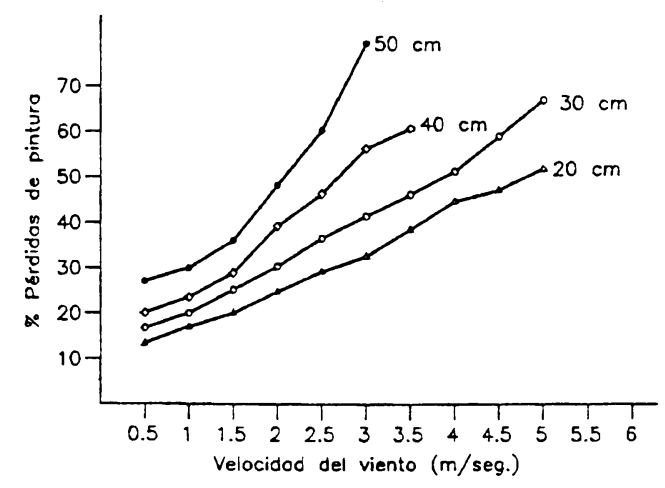

Pérdidas de pintura estimadas a distintas velocidades del viento $y$ diferentes distancias de la boquilla al soporte. Aplicación a airless.

\section{Precauciones especiales}

. Especialmente en los alrededores de tuercas y remaches, cantos vivos, perfiles pequeños, etc.; los espesores suelen ser irregulares, lo que se traduce en un mayor consumo de pintura.

. Zonas de difícil acceso o con dificultades de aplicación, disminuyen el rendimiento de la pintura por la imposibilidad de repartirla uniformemente.

. En pintados al exterior, el consumo de pintura está muy influenciado por las temperaturas extremas, frío o calor. La aplicación a brocha y rodillo comporta, generalmente, menos pérdidas que a pistola. El viento influye particularmente en el pintado a pistola airless.

\begin{tabular}{|cc|}
\hline $\begin{array}{c}\text { Perfil } \\
\text { en micrómetros }\end{array}$ & $\begin{array}{c}\text { «Volumen muerto » } \\
\text { en } \mathbf{c m}^{3} / \mathbf{m}^{2}\end{array}$ \\
\hline 10 & 6,66 \\
30 & 20,00 \\
50 & 33,33 \\
75 & 50,00 \\
100 & 66,66 \\
150 & 100,00 \\
200 & 133,32 \\
\hline
\end{tabular}


Factor de rugosidad $\left(\mathrm{f}_{\mathrm{r}}\right)$

\begin{tabular}{|l|c|c|}
\hline \multirow{2}{*}{ TIPOS DE SUPERFICIE } & \multicolumn{2}{|c|}{ Factor de rugosidad $\left(\mathbf{f}_{\mathrm{r}}\right)$} \\
\cline { 2 - 3 } & $\begin{array}{c}\text { Primeras } \\
\text { capas }\end{array}$ & $\begin{array}{c}\text { Capas } \\
\text { intermedias } \mathbf{y} \\
\text { de acabado }\end{array}$ \\
\hline - Acero liso & 0,95 & 0,98 \\
- Acero nuevo chorreado & 0,8 & 0,95 \\
- Acero preparado con limpieza \\
$\begin{array}{l}\text { mecánico/manual o chorreado } \\
\text { ligeramente picado. }\end{array}$
\end{tabular}

Factor de aplicación $\left(f_{a}\right)$

\begin{tabular}{|l|c|}
\hline \multicolumn{1}{|c|}{ SISTEMAS DE APLICACIÓN } & Factor de aplicación $\left(f_{a}\right)$ \\
\hline - Brocha y rodillo. & $0,8-0,7$ \\
- Pistola en interiores sin viento. & $0,7-0,6$ \\
- Pistola en exteriores con viento. & $0,6-0,5$ \\
\hline
\end{tabular}




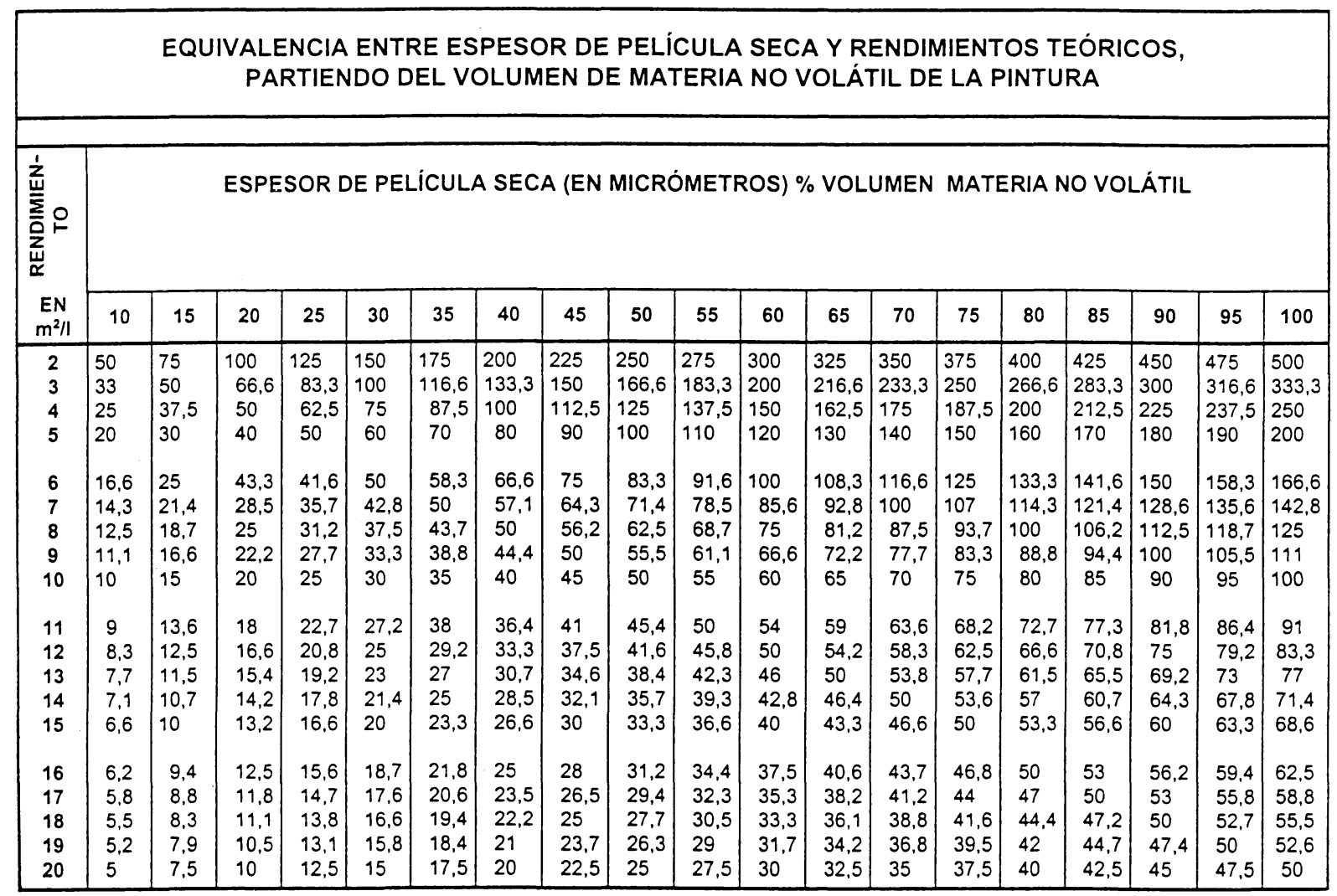

(Con este cuadro podremos conocer el espesor aproximado de pelicula después de seca.).

\section{Conclusión}

Un buen diseño del soporte, la correcta aplicación de la pintura y un mantenimiento adecuado, facilita, de manera notable, la realización de los trabajos, repercutiendo en el resultado final de la protección.

Para conseguir los mejores resultados al utilizar la pintura como un recubrimiento inhibidor de corrosión en las estructuras de acero, habría que tener en cuenta: $1^{\circ}$.- La correcta preparación del soporte,

$2^{\circ}$.- El tipo adecuado de imprimación elegido y su correcta aplicación

$3^{\circ}$.- La capa de recubrimiento elegida, compatible con la imprimación y su correcta aplicación.

$4^{\circ}$.- El fácil mantenimiento. 